\title{
On the Risk of Misbehaving RPKI Authorities
}

\author{
Danny Cooper ${ }^{*}$ Ethan Heilman* Kyle Brogle ${ }^{\dagger} \quad$ Leonid Reyzin $^{*} \quad$ Sharon Goldberg* \\ *Boston University, Boston, MA 02215 USA \\ †Stanford University, Stanford, CA 94305 USA \\ \{dannyc, heilman\}@bu. edu, broglek@stanford.edu, \{reyzin, goldbe\}@cs.bu . edu
}

\begin{abstract}
The RPKI is a new security infrastructure that relies on trusted authorities to prevent some of the most devastating attacks on interdomain routing. The threat model for the RPKI supposes that authorities are trusted and routing is under attack. Here we discuss the risks that arise when this threat model is flipped: when RPKI authorities are faulty, misconfigured, compromised, or compelled to misbehave. We show how design decisions that elegantly address the vulnerabilities in the original threat model have unexpected side effects in this flipped threat model. In particular, we show new targeted attacks that allow RPKI authorities, under certain conditions, to limit access to IP prefixes, and discuss the risk that transient RPKI faults can take IP prefixes offline. Our results suggest promising directions for future research, and have implications on the design of security architectures that are appropriate for the untrusted and error-prone Internet.
\end{abstract}

Categories \& Subject Descriptors. C.2.6 [ComputerCommunication Networks]: Internetworking

General Terms. Security, Standardization.

\section{INTRODUCTION}

A number of crucial Internet security infrastructures derive their security from information provided by authorities - trusted third parties who attest to information about cryptographic keys, domain names, and/or IP prefixes. Examples include DNS/DNSSEC; the public key infrastructure used for web (SSL/TLS) security; and, most recently, the RPKI 28], a new infrastructure for securing interdomain routing. When authorities behave correctly, each security infrastructure effectively prevents attacks on the system it was designed to protect 4, 8, 22. However, what happens if an authority

Permission to make digital or hard copies of all or part of this work for personal or classroom use is granted without fee provided that copies are not made or distributed for profit or commercial advantage and that copies bear this notice and the full citation on the first page. To copy otherwise, to republish, to post on servers or to redistribute to lists, requires prior specific permission and/or a fee.

Hotnets '13, November 21-22, 2013, College Park, MD, USA.

Copyright 2013 ACM 978-1-4503-2596-7 ...\$10.00.

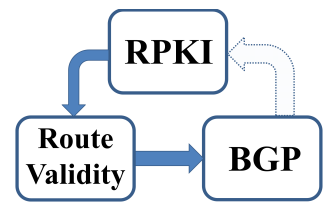

Figure 1: Dependencies.

malfunctions, is misconfigured, or is compromised by an external attacker? Centralized authorities are also an easy target for lawful (or extralegal) coercion by statesponsored actors seeking to impose censorship, information control, or surveillance. As state-sponsored interference in Internet systems has become more common in recent years $15,37,41]$, questions of Internet security also begin to have implications on Internet freedom.

We study the RPKI to gain insight on open questions related to the design of network security architectures that are robust to errors, misconfigurations, and abuse by authorities. This analysis is particularly timely given the recent problems with authorities in established systems like DNS and the web PKI; indeed there is ample evidence of authorities in both systems being hacked [5 17,31], misconfigured [45], or compelled by government agencies to delete information (e.g., DNS takedowns 37]) or to attest to bogus information 41]. We discuss how the RPKI presents a new point in the design space, show how its design creates unexpected side effects when authorities are compromised, and raise open problems with implications on the design of future Internet security infrastructures.

The RPKI. The RPKI 28] is a security infrastructure built on top of interdomain routing that has recently been standardized by the IETF and adopted by the Regional Internet Registries (RIRs). It is slowly being rolled out by individual network operators. The purpose of the RPKI is to provide a trusted mapping from an IP prefix to a set of autonomous systems (ASes) that are authorized to originate (i.e., claim to be the destination for) this prefix in interdomain routing. This trusted mapping can then be used to protect against the most devastating attacks on interdomain routing with BGP; namely, prefix and subprefix hijacks [8], where an AS originates ("hijacks") routes for IP prefixes that it is not 


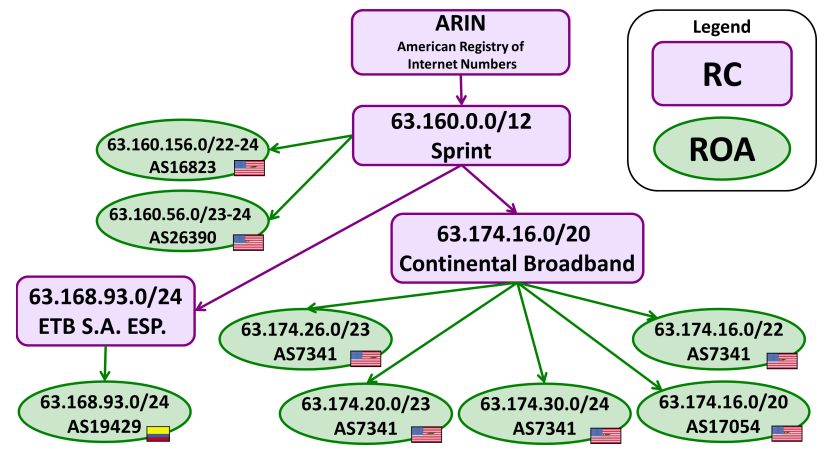

Figure 2: Excerpt of a model RPKI

authorized to originate, causing the traffic intended for those prefixes to be intercepted by the hijacker's network. As shown in Figure 1 information in the RPKI determines whether a route is valid, which can, in turn, determine the routes selected in BGP.

The RPKI is the necessary prerequisite for many more advanced proposals for securing BGP (e.g., 24, 27, 44)). Moreover, almost all of the routing attacks seen in the wild (e.g., 13, 32, 40 ) could be prevented if Internet routers dropped routes that the RPKI deems invalid; dropping RPKI-invalid routes is also surprisingly effective against more advanced routing attacks, even those that the RPKI was not designed to prevent [18,29].

A question. The potential for faulty or compromised RPKI authorities to instantaneously affect BGP routing has lead to some concern among practitioners and

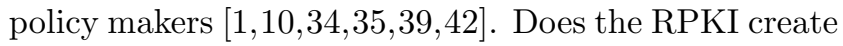
new risks that can take IP prefixes offline?

Our answer. One might expect this question to be completely addressed by the RPKI specifications. However, the RPKI is designed to operate in a threat model where authorities are trusted, but BGP is under attack. We therefore address operational and policy concerns by flipping the threat model: what if RPKI authorities are faulty, misconfigured, compromised, or coerced into behaving incorrectly? In Sections 3,4, we show how design decisions that elegantly address the vulnerabilities in the original threat model have unexpected side effects when analyzed in this flipped threat model.

The scope and variety of these threats is quite different than in a typical PKI. Section 3.1 shows how the hierarchical structure of the RPKI allows abusive authorities to exercise targeted control over their distant descendants (rather than just the objects they issue directly, as in a typical PKI). Section 4 shows how design decisions that are essential to preventing to subprefix hijacks on BGP mean that routing can be harmed if RPKI objects are simply missing (rather than revoked, corrupted, or forged, as in a typical PKI). We also close the loop in Figure 1 by showing how side effects can interact in a circular manner that can turn transient faults into persistent problems (Section 6). Finally, we discuss why (a) robustness to threats to BGP, and (b) robustness to threats to the RPKI, may be at odds (Section 5p; the risk that an RPKI problem can take a prefix offline therefore depends on the policies that routers use to balance the two threats against each other.

Organization. Section 2 overviews all components in Figure 1. Sections 3-6 analyze each individual component in the flipped threat model. Our results are based on measurement-driven models and analysis of RPKI software and RFCs (cited where appropriate throughout, along with related work).

To our knowledge, other research on the architecture of the RPKI is sparse, and covers network measurement 36, 43, and policy [10,34, 35, 42]. Our contributions are summarized in Section 7 .

\section{OVERVIEW: ROUTING WITH THE RPKI}

The RPKI. Most vulnerabilities in the web PKI result from architectural decisions that allow (almost) any authority to issue certificates for any subject 41$]$. In contrast, the RPKI follows the "principle of least privilege", arranging authorities in a strict hierarchy that mirrors the IP address allocation hierarchy. An authority may issue cryptographic objects for IP addresses that are covered by its own IP addresses 1 Today, IANA sits at the root of this hierarchy, allocating IP addresses to the Regional Internet Registries (RIRs), which allocate subsets of their address space to national/local internet registries (NIRs or LIRs) or ISPs, who further allocate subsets to other ISPs or customers ${ }^{2}$ In RPKI, each authority has a resource certificate $(R C)$ that contains its cryptographic public key and its set of allocated IP addresses [30. An authority may issue signed cryptographic objects for IP addresses covered by its allocation, specifically: (1) an RC that suballocates a subset of its addresses to another authority, or (2) a route origin authorization (ROA 3 that authorizes a specified AS to originate a prefix, and its subprefixes up to a specified length, in BGP [28, Section 2.2].

Figure 2 shows how an RIR (ARIN) uses its RC to suballocate a prefix to another authority (Sprint), which then issues RCs suballocating this prefix to other authorities (ETB S.A. ESP., Continental Broadband). We say Sprint is the parent of Continental Broadband, and extend this to child, grandparent, etc. in the obvious way. Sprint issues two ROAs that authorize specified prefix and its subprefixes of length up to 24; the remaining ROAs shown authorize only a single prefix.

\footnotetext{
${ }^{1}$ An IP prefix $P$ covers prefix $\pi$ if $\pi$ is a subset of the address space in $P$ (e.g., 63.160.0.0/12 covers 63.168.93.0/24) or if $P=\pi$. Also, a prefix 63.160.0.0/12 has length 12 .

${ }^{2}$ The root(s) of the RPKI hierarchy are not yet specified, but will likely be the five RIRs or IANA 28 , Section 2.4].

${ }^{3}$ Strictly speaking, an authority issues a one-time-use endentity (EE) certificate, which is then used to sign the ROA, but that detail is not important for this paper.
} 
Route validity (Section 4.) A relying party is a party that uses information in the RPKI to make routing decisions in BGP. For our purposes, a BGP route is an IP prefix and an origin AS. RPKI objects are stored in publicly-available repositories distributed throughout the Internet. Once a relying party has "access to a local cache of the complete set of valid ROAs" 20, Sec. 2], these valid ROAs are used to classify each route learned in BGP into one of three route validation states. Routes with matching valid ROAs are classified as valid. Other routes are either invalid or unknown. The RPKI allows arbitrary prefix lengths, but the smallest IPv4 prefix length which is globally routable in BGP is a /24; so the presence or absence of finer-grained RCs and ROAs has little impact on BGP.

BGP (Section 5.) A relying party uses a route's validation state to decide what routes to select in BGP. What impact does an invalid (or unknown) route have on BGP? This depends on "local policies" at each relying party 20 that reflect tradeoffs between robustness to RPKI attacks and robustness to BGP attacks.

\section{MANIPULATIONS OF THE RPKI}

Recall that a route is authorized by using ROA. Here we show how the architecture of the RPKI's certificate hierarchy enables targeted manipulations that can cause ROAs to become invalid. Sections 4 . 5 discuss the impact of an invalid ROA on BGP routing.

Design Decision: Revocation. In a traditional PKI, an authority can revoke any child certificate it issued, to remedy compromises of its child's cryptographic keys 12 . The RPKI inherits this functionality.

Side Effect 1: Unilateral reclamation of IP address allocations, with little recourse. Revocation of RCs or ROAs in the RPKI creates a new technical mechanism for an authority to unilaterally reclaim IP address space. Extending Amante's apt comparison of an RIR to a registry of deeds [1] for real estate, we can think of RPKI as a system of leases and subleases of IP addresses. RPKI design gives a landlord unilateral power to evict a tenant with whom it may have a business dispute or a political disagreement. This creates precisely the imbalance of power that eviction laws try to correct. The RPKI's hierarchical nature also means that the holder of the reclaimed space has little recourse available, since its space may only be reissued by authorities holding supersets of the reclaimed space (similar to DNS but in stark contrast with the web PKI, where any authority may issue any certificate).

Revocation is typically done via a CRL, a publiclyavailable list of revoked certificates that is signed by the revoking authority 12 . Relying parties could use this list to detect and react to abusive revocations. However, we now show that other design decisions allow RPKI objects to be revoked in a less transparent manner.
Design Decision: Distributed RPKI repositories and out-of-band certificate delivery. In the traditional PKI, the subject of the certificate delivers it to the verifier [26, p. 40]; a website sends its web certificate to a client in an SSL/TLS handshake. In contrast, BGP lacks a handshake phase, and the RPKI was designed to require minimal changes to BGP. In RPKI, relying parties download and verify RPKI objects out of band (rather in real time as part of BGP), and RPKI objects are stored at directories that are controlled by their issuer 19 28, Section 8]. For example, the two RCs and two ROAs issued by Sprint in Figure 2 are held by entities other than Sprint but are published by Sprint at a directory controlled by Sprint. In this sense, the RPKI is more similar to a trusted directory (e.g., DNS) than to a traditional PKI.

Design Decision: Objects can be overwritten. An RPKI authority may overwrite RCs and ROAs that it issued, so that objects can have persistent names (which simplifies operations like key rollover 21]).

Side Effect 2: Stealthy revocation of a child's object. Therefore, an authority can delete any ROA or $\mathrm{RC}$ it issued from its repository [23, or even overwrite it with one for a smaller set of IP addresses. This complicates attempts to monitor the RPKI for abusive revocations, especially since distinguishing between abusive behavior and normal RPKI churn could be difficult.

We now present new attacks that can make a ROA invalid in the RPKI. To unify terminology, we say that an RPKI manipulator whacks a target $\mathrm{ROA}$, regardless whether this is accomplished by a known method above or by a new method below.

\subsection{Targeted whacking of distant descendants.}

Revocation is a blunt instrument in a hierarchical PKI, as it invalidates an entire subtree of certificates, causing obvious and undesirable damage. For example, if Sprint wanted to target the ROA (63.174.16.0/20, 17054) in Figure 2, it could revoke the RC issued to Continental Broadband, but this would whack four additional ROAs as collateral damage; one might argue that the outcry from this collateral damage could deter deliberate revocations 38. However, we show that an RPKI manipulator can exercise fine-grained control over ROAs that are its distant descendants without whacking other ROAs as collateral damage.

Design Decision: Fine-grained resource allocation. In a traditional PKI, an authority binds a single name to a cryptographic key. By contrast, RPKI authorities bind arbitrary sets of IP addresses to a key.

Side Effect 3: Targeted whacking of a grandchild. Because an authority may issue RCs for arbitrary subsets of its IP addresses, a manipulator can whack any grandchild ROAs by removing, from the target's parent $\mathrm{RC}$, a portion of the address space con- 


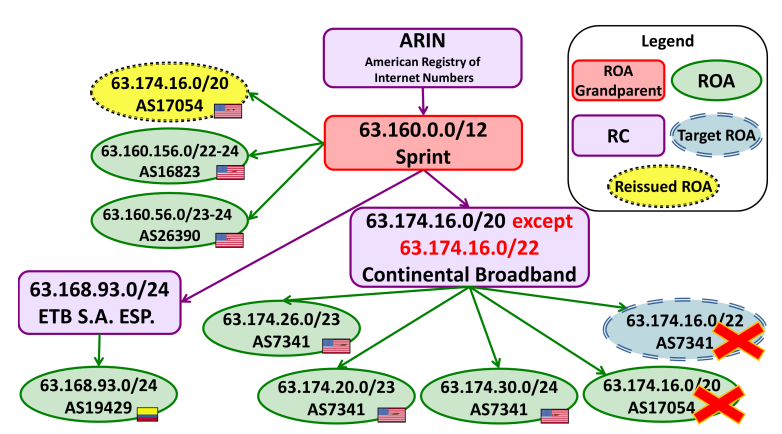

Figure 3: A ROA whacked by its grandparent.

tained in the target ROA. If the removed portion of the space overlaps no other RCs or ROAs issued by the target's parent, this action will cause no collateral damage. For example, Sprint can surreptitiously the target ROA $(63.174 .16 .0 / 20,17054)$ in Figure 2 by overwriting the RC it issued for Continental Broadband with the one for the two IP ranges [63.174.16.0-63.174.23.255] and [63.174.25.0-63.174.31.255]. Because this new RC covers all the ROAs issued by Continental Broadband (except the target ROA), all other ROAs remain valid.

When non-overlapping space cannot be found (e.g., if Sprint wants to target the ROA (63.17.16.0/22, AS 7341) in Figure 2), the manipulator can first (1) reissue the damaged descendant objects as its own ("makebefore-break"), and then (2) overwrite the appropriate child RC (as shown in Figure 3). This situation is easier to detect, due to the suspiciously-reissued ROA. One of the open problems we are working is the design of monitoring schemes that deter RPKI manipulations by detecting suspiciously reissued objects.

Side Effect 4: Whacking of great-grandchildren and beyond. ROAs below grandchild level can also be whacked without collateral damage. However, details of how RPKI objects point to RPKI repositories mean that this whacking requires more suspiciouslyreissued objects, and could be easier to detect. Our technical report [11] has the gory details.

\subsection{International borders.}

When a manipulator whacks a ROA in the same legal jurisdiction, the holder of the target ROAs may have some legal recourse against the manipulator's action. But what if the manipulator and target are in different jurisdictions? Indeed, many IPv4 addresses were historically suballocated with little regard for questions of international jurisdiction. Using BGP data, information about IP address allocations, and AS-to-country mappings provided by the RIR\&4 (details in our technical report 11) we found that cross-country certification is not uncommon. RIRs can whack ROAs for

\footnotetext{
${ }^{4}$ We use this data because today's production RPKI deployment is too small-about 1200-1400 ROAs, less than $1 \%$ of projected deployment according to our models and 36 .
}

\begin{tabular}{|lr||l|}
\hline Holder & RC & Countries \\
\hline Level3 & $8.0 .0 .0 / 8$ & RU,FR,NL,CN,TW,JP,GU,AU,GB,MX \\
Cogent & $38.0 .0 .0 / 8$ & GU,GT,HK,GB,IN,PH,MX \\
Verizon & $65.192 .0 .0 / 11$ & CO,IT,AN,AS,GB,EU,SG \\
Sprint & $208.0 .0 .0 / 11$ & AS,BO,CO,ES,EC \\
Sprint & $63.160 .0 .0 / 12$ & FR,CO,YE,AN,HN \\
Tata Comm. & $64.86 .0 .0 / 16$ & GU,CO,MH,HN,PH,ZW \\
Columbus & $63.245 .0 .0 / 17$ & NI,GT,CO,AN,HN,MX \\
Servcorp & $61.28 .192 .0 / 19$ & FR,AE,CA,US,GB \\
Resilans & $192.71 .0 .0 / 16$ & US,IN \\
\hline
\end{tabular}

Table 4: A few RCs \& the countries they cover that are outside jurisdiction of their parent RIR.

ASes in non-member countries, even though they are are accountable only to their member countries. For example, through its certification of Sprint, North America's ARIN can whack ROAs for Europe and the Middle East. Europe-based RIPE can whack ROAs in Asia and the Americas. A few RCs held by private entities also cover ROAs in multiple countries. Table 4 has a few salient examples.

\section{4. $\quad$ RPKI $\Rightarrow$ ROUTE VALIDITY}

Route validity decisions are made by relying parties once they have determined a complete set of all valid ROAs and stored them in a local cache 20, Sec. 2]. If a ROA is whacked, expires, or is missing due to a fault or misconfiguration, it will not be in this local cache. What impact does its absence have on route validity? We show how the semantics of determining route validity, which were designed to limit the risk of subprefix hijacks on BGP, can lead to unintuitive consequences.

Design Decision: Retaining BGP's subprefix semantics. BGP is vulnerable to subprefix hijacks because of longest-prefix-match routing: when a router is offered BGP routes for a prefix and its subprefix, it always chooses the subprefix route. Subprefix hijackers exploit this by originating routes for subprefixes of a victim prefix [40. This leads to a natural design goal: a subprefix hijacker's route should be invalid when victim's route has a matching valid ROA. To achieve this goal, a relying party performs origin authentication as follows. Each BGP route for prefix $\pi$ and origin AS $a$ is classified with one of three validation states, based on all the valid ROAs in the relying party's local cache 20,33]:

- Valid: There is a valid matching ROA. A matching ROA has (1) a matching origin AS $a$, and (2) a prefix $P$ that covers prefix $\pi$, and (3) the specified maximum length no shorter than the length of $\pi$.

- Unknown: There is no valid covering ROA. A covering ROA is any ROA for a prefix that covers $\pi$.

- Invalid: The route is neither unknown or valid.

Figure 5 (left) shows how the ROAs in Figure 2 determine the validity of routes for 63.160.0.0/12 and all its subprefixes. The rules above elegantly achieve the design goal; the ROA for (63.174.16.0/20, AS 17054) protects the corresponding route from subprefixes hi- 

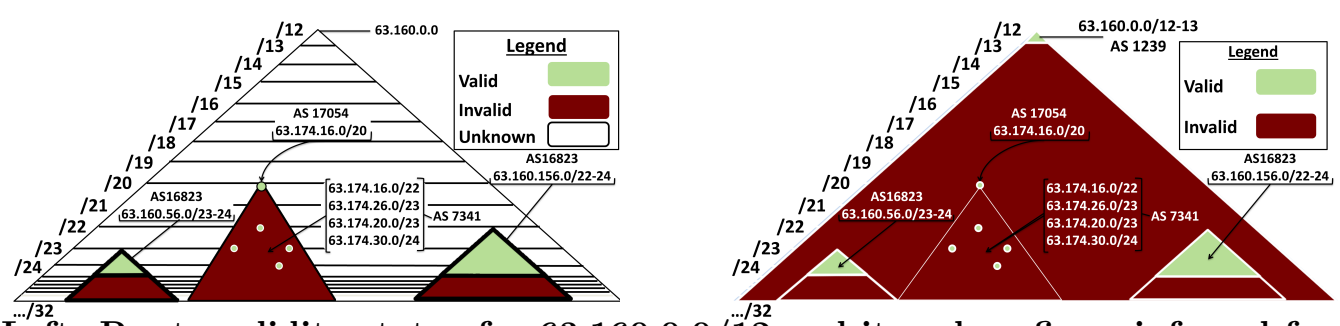

Figure 5: $\quad$ Left: Route validity status for $63.160 .0 .0 / 1 / 32$ and its subprefixes, inferred from the RPKI of Figure 2, Right: The change in route validity if a ROA (63.160.0.0/12-13, AS1239) is added.

jacks, because all routes for its subprefixes are "invalid" (except routes with matching ROAs of their own).

These rules also imply that route is "unknown" only if there is no covering $R O A$ t The RPKI in Figure 2 contain ROAs for neither 63.160.0.0/12 nor 63.174.17.0/24. However, Figure 5 (left) shows that routes for 63.160.0.0/12 are "unknown" (there is no covering ROA) but routes for 63.174.17.0/24 are "invalid" (because of the ROA for $63.174 .16 .0 / 20)$. This has two side effects.

Side Effect 5: A new ROA can cause many routes to become invalid. Figure 5 (right) shows that if Sprint issues a new ROA (63.160.0.0/12-13, AS 1239) that covers previously unknown routes, those routes become "invalid". This creates a deployment challenge, since (a) the earliest adopters of the RPKI are likely to be large networks (like Sprint) that hold large prefixes, but (b) a new ROA for a large prefix should be issued only after all ROAs for its subprefixes, to prevent routes from mistakenly becoming invalid. Indeed, 43 found that the production RPKI classified many production BGP routes as invalid, likely for this very reason.

Side Effect 6: A missing ROA can cause a route to become invalid. Missing information is problematic in any secure system, especially so in the RPKI, because the absence of a ROA in a relying party's local cache does not mean that the corresponding route is merely "unknown" (as in e.g., DNSSEC or the web $\mathrm{PKI}$ ). The requirement that relying parties have access to a complete set of valid ROAs [20, Sec. 2] is therefore crucial; for example, if the ROA (63.174.16.0/22, AS 7341) is missing from the RPKI of Figure 2 the corresponding route will be classified as "invalid" (instead of "unknown") because of the covering ROA for prefix 63.174.16.0/20 (see Figure 5(left)). This makes the RPKI vulnerable to faults that disrupt the delivery of valid ROAs, a side effect that is easily misunderstood 3 14. Information can be missing for a variety of reasons: the renewal of an expiring ROA could be delayed (accidentally or maliciously); the filesystem or server storing the ROA could become corrupted; etc.. While this may cause only temporary disruptions, Section 6 discusses how this can create persistent failures.

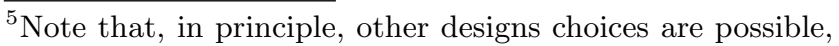
e.g., requiring each ROA to explicitly indicate which routes for its subprefixes should remain valid or unknown.
}

\begin{tabular}{|c||c|c|}
\hline \multicolumn{1}{|c||}{ relying-party } & \multicolumn{2}{c|}{ Prefix reachable during... } \\
policy & routing attack & RPKI manipulat'n \\
\hline \hline drop invalid & $\checkmark$ & $X$ \\
\hline depref invalid & subprefix hijacks possible & $\checkmark$ \\
\hline
\end{tabular}

Table 6: Impact of different local policies.

A difficult tradeoff: What to do about incomplete information? The RFCs do not specify what action should be taken when a relying party suspects a valid ROA might be missing from a repository (e.g., see [2, Sect 6.5]). Should a party stop relying on the RPKI if it thinks ROAs could be missing? On one hand, this avoids the problems discussed in Side Effect 6. On the other, it opens up the relying party to BGP attacks.

It is an open problem to design architectures for route validity that prevent subprefix hijacks but are not brittle in case of missing information or misconfiguration. Alternatively, monitoring and configuration tools could be used to mitigate these risks.

Summary: RPKI problems $\Rightarrow$ invalid routes? We have seen that a route can become "invalid" due to: (1) a misconfiguration by an RPKI authority (Side Effect 5), (2) missing information in a relying party's cache (Side Effect 6), (3) a ROA that is whacked AND is also covered by a valid ROA (Section 3 ).

\section{ROUTE VALIDITY $\Rightarrow$ BGP}

What impact does an invalid (or unknown) route have on BGP routing? That depends on to the "local policies" at each relying party [20. We now consider the two most plausible policies, as suggested by 20]:

Drop Invalid: This policy requires that a relying party never selects an invalid route. It fully realizes RPKI's potential to protect routing, stopping prefix and subprefix hijacks (Section 1). However, if RPKI problems causes a route to become invalid, the relying party will lose connectivity to the corresponding IP prefix.

Depref invalid: This (more lenient) policy requires that, for a given prefix, a router prefers valid routes over invalid routes. This means that a router still selects an invalid route when there is no valid route for the exact same IP prefix. Thus, the router may still be able to reach prefixes whose routes have become invalid due to problems with the RPKI. However, this policy does not prevent subprefix hijacks; see [6, Section 5]. 
A difficult tradeoff: RPKI attacks vs. BGP attacks? Table 6 highlights a tradeoff that is implicit in the RPKI RFCs; namely, that the local policy that is best at protecting against problems with BGP is worst at protecting against problems with RPKI. Balancing these considerations is a challenging open problem.

\section{CLOSING THE LOOP: BGP $\Rightarrow$ THE RPKI}

Finally, we highlight the complexities involved in architecting a system like the RPKI, by closing the loop in Figure 1. To do this, we show how a chain of (unlikely, but plausible) events can lead to persistent failures.

Design Decision: Delivery of RPKI information over TCP/IP. A PKI is typically deployed as a layer on top of a (possibly unauthenticated) communication infrastructure; web (HTTPS) certificates, for example, are delivered over TCP/IP. Similarly, the only delivery method mandated by the RPKI is the rsync protocol 19, Section 2.2], which runs on top of TCP/IP. (Other delivery methods are allowed at operator discretion.) However, unlike web certificates, RPKI objects can affect the availability of BGP routes, and therefore also of TCP/IP, the very infrastructure over which they are delivered. This can create a circular dependency.

Side Effect 7: Transient faults cause long-term failures. We now show how this design decision, the decision to allow distributed RPKI repositories located anywhere in the Internet (Section 3), and the issues in Sections 4 4.5. can cause a transient error to become a persistent failure. Suppose that (1) route validity is as shown in Figure 5 (right), (2) Continental Broadband (AS 17054) hosts its own repository at 63.174.23.0, and (3) a relying party drops invalid routes in BGP.

This example contains a circularity: for the relying party to retrieve ROAs issued by Continental Broadband, it must have a valid or unknown route to Continental Broadband's repository at 63.174.23.0 and AS 17054. Because route validity is as in Figure 5 (right), the route to the repository will be invalid unless the relying party can retrieve the ROA binding 63.174.16.0/20 to AS 17054. However, this ROA is issued by Continental Broadband, and is therefore hosted at Continental Broadband's repository. Thus, for the relying party to access Continental Broadband's repository, it must first access a ROA that is stored at that repository.

Now suppose a transient error causes the relying party to receive a corrupted ROA for $(63.174 .16 .0 / 20$, AS 17054) (see Side Effect 6). As explained above, the relying party will lose access to Continental Broadband's repository. Even if the fault is remedied and the repository is ready to serve the missing ROA, the relying party cannot obtain the missing ROA, because it cannot reach the repository. This can be fixed (manually), but there no are recommended procedures for recovery.
The example arises because (a) the ROA for a route to an RPKI repository is stored at that same repository, and (b) another ROA covers but does not match the route to the repository, and (c) the relying party drops invalid routes. (Condition (a), but not its implications, was also pointed out by [20].) More complex circular dependencies can exist, involving multiple ROAs and repositories, and it is an open question to develop operational guidelines that eliminates these dependencies.

\section{CONCLUSION \& OPEN PROBLEMS}

The RPKI has the potential to eliminate most of the routing attacks seen in the wild (e.g., $13,32,40]$ ), and is a prerequisite for more advanced proposals for securing BGP 8. However, we showed that its architecture creates new technical means for authorities to unilaterally reclaim IP address allocations (Side Effects 1 22, in a targeted manner, even to distant descendants (Side Effects 3 4). This leaves the target with little recourse, especially when the relationship between the target and authority crosses international borders (Section 3.2). We note that these manipulations are more coarse-grained than domain name seizures [38, because current BGP practices limit their granularity to a $/ 24$ IPv4 prefix, i.e., $256 \mathrm{IPv} 4$ addresses.

We also showed how confusion (Side Effect 5 ) and sensitivity to missing information (Side Effect 6) can lead to RPKI misconfigurations that cause routes to become invalid. Finally, we showed how circular dependencies between the RPKI and BGP can lead to persistent failures (Side Effect 7). Our results leave RPKI relying parties with a seemingly difficult tradeoff (Section 5): They can use local policies that (a) send traffic on invalid routes, thus reducing their vulnerability to problems with the RPKI while increasing vulnerability to problems with BGP, or (b) stop sending traffic on invalid routes, which has the opposite effect.

Open Problems. The routing security improvements promised by the RPKI 8, 18, 29] motivate efforts to harden the RPKI against errors, misconfigurations, and abuse; indeed, concurrently to our work there have been new steps in this direction in the IETF $7,16,25]$. There are a number of issues to address. Can abuse by RPKI authorities be made more difficult to execute, more limited in scope, or easier to detect? Is the RPKI's sensitivity to missing objects caused by fundamental design requirements, or are there alternate architectures that are more robust? Can we develop better local policies for relying parties that overcome the difficult tradeoff we mentioned above? How should Internet routing be secured if the only means of communication is the Internet itself? Addressing these issues in the context of the RPKI will also inform the design of future security architectures that are appropriate for the inherently untrusted and error-prone Internet. 
Acknowledgements. We thank Steve Kent for suggesting the term "ROA whacking" to us, Tony Tauber for useful discussions, FCC CSRIC Working Group 6 - Secure BGP Deployment for inspiring this study, CAIDA for early access to their AS to organization mapping 9], and Dimitris Papadopoulos, Davide Proserpio, and Jennifer Rexford for comments on this draft. This work was supported by NSF awards 1017907, 1012798, and 1012910.

\section{REFERENCES}

[1] S. Amante. Risks associated with resource certification systems for internet numbers, 2012.

[2] R. Austein, G. Huston, S. Kent, and M. Lepinski. RFC 6486: Manifests for the Resource Public Key Infrastructure (RPKI). Internet Engineering Task Force (IETF), 2012 http://tools.ietf.org/html/rfc6486

[3] A. Band. "Re: rpki vs. secure dns?", msg566. seclists NANOG Archive, apr 2012. http://seclists.org/nanog/2012/Apr/566

[4] M. Benantar. The internet public key infrastructure. $I B M$ Systems Journal, 40(3):648-665, 2001.

[5] P. Bright. arstechnica: How the Comodo certificate fraud calls CA trust into question, March 2011. http://arstechnica.com/security/2011/03/how-the-comodocertificate-fraud-calls-ca-trust-into-question/

[6] R. Bush. RPKI-Based Origin Validation Operation. Internet Engineering Task Force Network Working Group, 2012. http://tools.ietf.org/html/draft-ietf-sidr-origin-ops-19

[7] R. Bush. RPKI Local Trust Anchor Use Cases. Internet Engineering Task Force (IETF), 2013. http://www.ietf.org/id/draft-ymbk-lta-use-cases-00.txt

[8] K. Butler, T. Farley, P. McDaniel, and J. Rexford. A survey of BGP security issues and solutions. Proceedings of the IEEE, 2010.

[9] CAIDA. AS to organization mapping. http://as-rank.caida.org/?mode0=as-intro\#as-org

[10] Communications Security, Reliability and Interoperability Council III (CSRIC). Secure bgp deployment. Communications and Strategies.

[11] D. Cooper, E. Heilman, K. Brogle, L. Reyzin, and S. Goldberg. On the risk of misbehaving RPKI authorities. Technical report Boston University, 2013.

[12] D. Cooper, S. Santesson, S. Farrell, S. Boeyen, R. Housley, and W. Polk. RFC 5280: Internet X.509 Public Key Infrastructure Certificate and Certificate Revocation List (CRL) Profile. Internet Engineering Task Force (IETF), 2008. http://tools.ietf.org/html/rfc5280

[13] J. Cowie. Rensys blog: China's 18-minute mystery. http://www. renesys.com/blog/2010/11/chinas-18-minute-mystery.shtml

[14] J. Curran. "Re: [sidr| Princeton University:: Impacting IP Address Reachability via RPKI Manipulations", msg05906. IETF, sidr archive, apr 2013. http:

//www.ietf.org/mail-archive/web/sidr/current/msg05906.html

[15] R. Deibert, J. Palfrey, R. Rohozinski, and J. Zittrain. Access controlled: The shaping of power, rights, and rule in cyberspace. MIT Press, 2010.

[16] R. Gagliano, T. Manderson, and C. M. Cagnazzo. Multiple Repository Publication Points support in the Resource Public Key Infrastructure $(R P K I)$. Internet Engineering Task Force (IETF), 2013. http://tools.ietf.org/html/ draft-ietf-sidr-multiple-publication-points-00

[17] E. Galperin, S. Schoen, and P. Eckersley. A post mortem on the iranian diginotar attack. EFF Blog, September 2011.

[18] S. Goldberg, M. Schapira, P. Hummon, and J. Rexford. How secure are secure interdomain routing protocols? In SIGCOMM'10, 2010

[19] G. Huston, R. Loomans, and G. Michaelson. RFC 6481: A Profile for Resource Certificate Repository Structure. Internet Engineering Task Force (IETF), 2012. http://tools.ietf.org/html/rfc6481

[20] G. Huston and G. Michaelson. RFC 6483: Validation of Route Origination Using the Resource Certificate Public Key Infrastructure (PKI) and Route Origin Authorizations (ROAs). Internet Engineering Task Force (IETF), 2012. http://tools.ietf.org/html/rfc6483

[21] G. Huston, G. Michaelson, and S. Kent. RFC 6489 Certification Authority (CA) Key Rollover in the Resource Public Key Infrastructure (RPKI). Internet Engineering Task Force (IETF), 2012. http://tools.ietf.org/html/rfc6489

[22] D. Kaminsky. Black ops 2008: Its the end of the cache as we know it. Black Hat USA, 2008.
[23] S. Kent and A. Chi. Rfc draft: Threat model for bgp path security. 2013 http://tools.ietf.org/html/draft-kent-bgpsec-threats-01

[24] S. Kent, C. Lynn, and K. Seo. Secure border gateway protocol (S-BGP). J. Selected Areas in Communications, 18(4):582-592, April 2000.

[25] S. Kent and D. Mandelberg. Suspenders: A Fail-safe Mechanism for the RPKI. Internet Engineering Task Force (IETF), 2013. http://tools.ietf.org/html/draft-kent-sidr-suspenders-00

[26] L. M. Kohnfelder. Towards a Practical Public-key Cryposystem. Massachusetts Institute of Technology, 1978. Bachelor's Thesis. http://groups.csail.mit.edu/cis/theses/kohnfelder-bs.pdf

[27] M. Lepinski, editor. BGPSEC Protocol Specification. IETF Network Working Group, Internet-Draft, July 2012. Available from http:
//tools.ietf .org/html/draft-ietf-sidr-bgpsec-protocol-04

[28] M. Lepinski and S. Kent. RFC 6480: An Infrastructure to Support Secure Internet Routing. Internet Engineering Task Force (IETF), 2012. http://tools.ietf.org/html/rfc6480

[29] R. Lychev, S. Goldberg, and M. Schapira. Is the juice worth the squeeze? BGP security in partial deployment. In SIGCOMM'13, 2013.

[30] T. Manderson, L. Vegoda, and S. Kent. RFC 6491: Resource Public Key Infrastructure (RPKI) Objects Issued by IANA". Internet Engineering Task Force (IETF), 1973. http://tools.ietf.org/html/rfc6491

[31] M. Marquis-Boire. A brief history of dns hijackings (at google). ICANN'43, March 2012

[32] S. Misel. "Wow, AS7007!". Merit NANOG Archive, apr 1997. Www.merit.edu/mail.archives/nanog/1997-04/msg00340.html

[33] P. Mohapatra, J. Scudder, D. Ward, R. Bush, and R. Austein. RFC 6811: BGP prefix origin validation. Internet Engineering Task Force (IETF), 2013. http://tools.ietf.org/html/rfc6811

[34] M. Mueller and B. Kuerbis. Negotiating a new governance hierarchy: An analysis of the conflicting incentives to secure internet routing. Communications and Strategies, (81):125-142, 2011

[35] M. Mueller, A. Schmidt, and B. Kuerbis. Internet security and networked governance in international relations. International Studies Review, 15(1):86-104, 2013.

[36] E. Osterweil, T. Manderson, R. White, and D. McPherson. Sizing estimates for a fully deployed rpki. Technical report, Verisign Labs Technical Report, 2012.

[37] D. Piscitello. Guidance for preparing domain name orders, seizures \& takedowns. Technical report, ICANN, March 2012

[38] D. Piscitello. The value of assessing collateral damage before requesting a domain seizure. Technical report, ICANN, January 2013.

[39] I. G. Project. In important case, RIPE-NCC seeks legal clarity on how it responds to foreign court orders, 2011. http://www.internetgovernance.org/2011/11/23/in-importantcase-ripe-ncc-seeks-legal-clarity-on-how-it-responds-to-foreigncourt-orders/

[40] Rensys Blog. Pakistan hijacks YouTube. http://www.renesys . com/blog/2008/02/pakistan_hijacks_youtube_1.shtml

[41] C. Soghoian and S. Stamm. Certified lies: Detecting and defeating government interception attacks against ssl (short paper). In Financial Cryptography and Data Security, pages 250-259. Springer, 2012.

[42] The President's National Security Telecommunications Advisory Committee. Nstac report to the president on communications resiliency, 2011.

[43] M. Wählisch, O. Maennel, and T. Schmidt. Towards detecting BGP route hijacking using the RPKI. In Poster: SIGCOMM'12, pages 103-104. ACM, 2012.

[44] R. White. Deployment considerations for secure origin BGP (soBGP). draft-white-sobgp-bgp-deployment-01.txt, June 2003, expired.

[45] C. Wisniewski. Turkish certificate authority screwup leads to attempted google impersonation. Naked Security Blog, January 42013. 